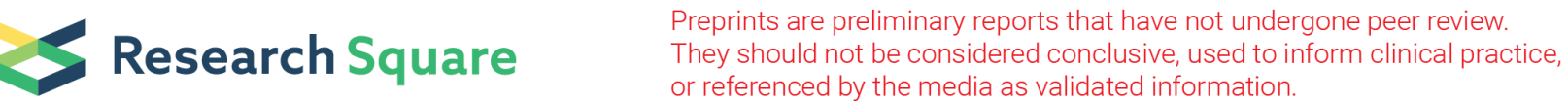

\section{Comparison between dynamic stabilization and instrumented fusion in the treatment of spinal stenosis with degenerative lumbar scoliosis}

Lei Luo

the Third Affiliated Hospital of Chongqing Medical University

Lang Fang

the Third Affiliated Hospital of Chongqing Medical University

Qiang Zhou ( $\square$ zhouqiang@hospital.cqmu.edu.cn )

The third affiliated hospital of chongqing medical university https://orcid.org/0000-0001-6253-329X

\section{Chen Zhao}

the Third Affiliated Hospital of Chongqing Medical University

Pei Li

the Third Affiliated Hospital of Chongqing Medical University

Liehua Liu

the Third Affiliated Hospital of Chongqing Medical University

Lichuan Liang

the Third Affiliated Hospital of Chongqing Medical University

Fei Luo

Southwest Hospital of Army Medical University

Yanhong Chen

the Third Affiliated Hospital of Chongqing Medical University

\section{Research article}

Keywords: Dynamic stabilization, Dynesys, Lumbar spine, Posterior instrumented stabilization, Degenerative lumbar scoliosis

Posted Date: December 30th, 2019

DOl: https://doi.org/10.21203/rs.2.19711/v1

License: (c) (i) This work is licensed under a Creative Commons Attribution 4.0 International License.

Read Full License 
Version of Record: A version of this preprint was published at Pain Research and Management on May 18th, 2022. See the published version at https://doi.org/10.1155/2022/9367106. 


\section{Abstract}

Background Posterior instrumented fusion is the most widely accepted surgical treatment for spinal stenosis with degenerative lumbar scoliosis (DLS). However, long fusion can affect daily activities due to lumbar stiffness. Several clinical studies have shown that Dynesys dynamic stabilization in addition to laminectomy could lead to significant improvements in clinical outcomes. This study aimed to compare the outcomes of Dynesys dynamic stabilization with posterior instrumented fusion for the management of spinal stenosis with DLS.

Methods Between August 2010 and Deccember 2015, a total of 46 patients with spinal stenosis and degenerative lumbar scoliosis were enrolled in this study. 26 patients (Dynesys group) had fenestration decompression, selective intervertebral fusion and Dynesys stabilizationin. 20 patients (fusion group) underwent posterior instrumented fusion. Clinical outcomes, radiographic data, and perioperative complications were compared between the two groups. The average duration of follow-up for the Dynesys group and fusion group was 38 and 33 months respectively.

Results The mean number of fixed segments were $3.3 \pm 0.8$ in Dynesys group and $4.3 \pm 0.8$ in fusion group. Lower average values of operative duration and blood loss were observed in the dynamic group than in the fusion group. VAS for back and leg pain improved in both groups of patients. There were significant difference in ODI and LSDI (lumbar stiffness disability index) between Dynesys group and fusion group at the last follow-up. The scoliosis Cobb's angle and lumbar lordosis significantly improved in both groups after surgery, and no significant difference were observed between the groups at the last follow-up ( $P$ 0.05). Dynesys stabilization resulted in significantly higher preservation of motion at the implanted segments.

Conclusions This study demonstrated that both Dynesys dynamic stabilization and instrumented fusion can improve clinical outcomes of patients with degenerative lumbar scoliosis. Compared to instrumented fusion, Dynesys stabilizationin have advantages on blood loss, operation time, perioperative complications. In addition, Dynesys stabilization partially preserves the ROM of the stabilized segments that may reduce the limitation on daily activities caused by lumbar stiffness. Dynesys stabilizationin can also correct scoliosis, prevent progression of the curve, and maintain lumbar lordosis in mild to moderate lumbar scoliosis without sagittal imbalance.

\section{Background}

Degenerative lumbar scoliosis(DLS) is defined as a spinal deformity which develops during adulthood due to asymmetric degenerative changes of the disc, vertebral body and facet joint,with a coronal Cobb measurement $\geq 10^{\circ}[1]$. It is a common disease in the middle-aged and elderly population[2]. In most patients with low back pain, the curve is likely to progress [3]. DLS is frequently associated with disc herniation, degenerative spondylolisthesis and stenosis [4]. The symptoms are mainly low back pain, radicular pain and neurogenic claudication. Patients with degenerative scoliosis often have some 
comorbidities such as hypertension, diabetes or respiratory diseases. So the treatment should focus on alleviating symptoms and preventing further progression of scoliosis, rather than deformity correction[5].

When conservative treatment fails, surgical treatment should be considered[6], At the present time, surgical treatments mainly include simple spinal decompression and lumbar fusion with instrumentation[7]. Simple spinal decompression can relieve radicular pain in the lower limbs. However, decompression alone presents a poor long-term result which is related to progression of deformity $[8,9]$. Lumbar fusion with instrumentation is the most widely accepted surgical treatment. It can be divided into short fusion and long fusion. Long fusion was superior to short fusion in the correction of the Cobb angle, coronal imbalance and lateral listhesis, but it is likely to increase perioperative complications [10]. In addition, long fusion can obviously affect the activities of lumbar spine, such as bending down, squatting, wiping after the stool, and so on [11].

In recent years, a dynamic stabilization system (Dynesys system, Fig. 1) has been used for DLS. Several clinical studies have shown that Dynesys dynamic stabilization in addition to laminectomy could lead to significant improvements in clinical outcomes and maintain enough stability to prevent progression of scoliosis and instability [12-15]. However, laminectomy damaged the posterior column structures of the lumbar spine which could increase the stress on the screws. Moreover, good lumbar lordosis was correlated with clinical symptoms[16], and significant lateral olisthesis could lead to scoliosis progression[3]. But Dynesys system has limited ability to correct lumbar kyphosis[17] and lateral olisthesis. Therefore, we treated DLS with fenestration decompression, selective intervertebral fusion and Dynesys dynamic stabilization. The purpose of this study is to discuss the clinical efficacy of this method and to evaluate its effectiveness in stabilizing the lumbar spine and preventing the progression of scoliosis.

\section{Methods}

\section{Patient Population}

Between August 2010 and Deccember 2015, a total of 46 patients with DLS were enrolled in this study (The inclusion and exclusion criteria are shown in Table 1). Patients were divided into 2 groups, Dynesys $(n=26)$ and instrumented fusion $(n=20)$. Five patients underwent additional single-segmental intervertebral fusion in the Dynesys group.

\section{Surgical procedure}

\section{Fenestration decompression, selective intervertebral fusion, dynamic stabilization with the Dynesys system.}

Patients were placed in the prone position under general anesthesia. Wiltse approach was used to expose the basilar part of the transverse process and the lateral margin of the superior articular[18]. The entry points of pedicle screws were located at the intersection of the lateral margin of the superior articular and 
the basilar part of the transverse process in the super - median $1 / 3$. Pedicle screws were positioned under imaging control. The extent of fixation included decompressed segments and segments with instability, spondylolisthesis, lateral olisthesis, not to end at the apical vertebra. Interlaminar fenestration was used for decompression. When there were segments recurvatum, lateral olisthesis $>12 \mathrm{~mm}$, or foraminal stenosis required removal of articular processes, transforaminal lumbar interbody fusion (TLIF) was performed. Then the patients' position was modified to obtain the appropriate lumbar lordosis. The polycarbonate urethane spacer was cut according the measured distance between the screws (distraction force $1.0 \mathrm{~N}$, longer on the concave side and shorter on the convex side). The spacer length was properly reduced in the fusion segment for intervertebral compression. The central cord and the spacer were then locked within the screw heads (Fig. 2). Autogenous blood transfusion was used in the operation. Patients received a soft support lumbar corset for 3 months after surgery.

\section{Posterior instrumented fusion}

Patients were placed in the prone position under general anesthesia. Posterior median approach was used to expose the lamina and facet joints. Pedicle screws were positioned under imaging control. Horizontal vertebra was selected as the upper instrumented vertebra. L5 or S1 was selected as the lower instrumented vertebra. Curve correction was carried out by distraction on the concave side and compression on the convex side. When there were segments need discectomy, or lateral olisthesis > $12 \mathrm{~mm}$, TLIF was performed. Posterolateral fusion was performed at other segments (Fig. 3). Autogenous blood transfusion was used in the operation. The patient wore a hard brace for 3 months after surgery.

\section{Clinical and Radiological Evaluation}

Clinical outcomes were assessed by means of visual analog scale (VAS) for back and leg pain, Oswestry Disability Index (ODI), and lumbar stiffness disability index [19] (LSDI, Table 2). Operative time, blood loss, and complications were also documented. Posteroanterior, lateral, and dynamic radiographs with flexion and extension views were obtained preoperatively, postoperatively, and at last follow-up. Radiological evaluation index included lumbar scoliotic angle, lumbar lordotic angle, and the range of motion (ROM). A "Double halo sign" (Radiolucent line aroud the implant $2 \mathrm{~mm}$ wide) on X-rays was defined as screw loosening.

\section{Statistical analysis}

SPSS 16.0 software was used for statistical analysis. Chi-square test and rank sum test were used for categorical data. T test was used for comparison among groups of quantitative data, and variance analysis and q test (student-newman-keuls, SNK) were used for intra-group comparison. If the condition of parameter test is not satisfied, the rank sum test method is adopted. When $P<0.05$, the difference was considered to be statistically significant.

\section{Results}




\section{Perioperative data and complications}

In the Dynesys group, the mean number of fixed segments was $3.3 \pm 0.8$. Mean operating time was $258 \pm 71$ minutes, while intraoperative blood loss was was $771 \pm 494 \mathrm{ml}$. One testicular hydrocele case, one oral mucous ulcer case, one upper respiratory infection case, and one pulmonary infection case were resolved after medical treatment. Poor wound healing occurred in 3 cases which was cured by secondary suture. One patient developed transitory radiating pain after surgery, which was relieved by medication. One patient developed muscle weakness of lower extremity, which was recovered by neurotrophic therapy and functional exercise 1 month after surgery. The mean follow-up duration was 38 months (range, 24-76 months). Screw loosening was found on plain radiographs in one patient. There were no cases of incision infection, screw misplacement, screw breakage, or reoperation.

In the Fusion group, the mean number of fixed segments was $4.3 \pm 0.8$. Mean operating time was $367 \pm 59$ minutes, while intraoperative blood loss was was $1280 \pm 538 \mathrm{ml}$. One atrial fibrillation case and one Deep venous thrombosis case were resolved after medical treatment. Poor wound healing occurred in 2 cases which was cured by secondary suture. One patient developed surgical site infection, which was cured by antibiotics and debridement. Two patient developed transitory radiating pain after surgery, which was relieved by medication. One patient developed severe low back pain, which was alleviated by symptomatic treatment with non-steroidal anti-inflammatory drugs (NAIDs). The mean follow-up duration was 33 months (range, 23-68 months). There were 3 cases of screw loosening, 1 case of proximal junctional kyphosis, and no cases of implant breakage or pseudarthrosis.

The difference in operation time and blood loss between the two groups was statistically significant $(\mathrm{P}<$ 0.05). The Dynesys group had shorter operation time and less blood loss.

\section{Clinical outcomes}

In the Dynesys group, the mean VAS scores for low back pain obtained preoperatively, 6 months after surgery and in the last follow-up visit were $5.2 \pm 1.7,2.3 \pm 1.4$, and $2.1 \pm 1.1$, respectively. The corresponding mean VAS scores for leg pain were $5.8 \pm 1.5,1.6 \pm 0.4$, and $1.8 \pm 0.9$. The corresponding ODI scores were $66.9 \pm 17.8,30.7 \pm 12.3$, and $21.6 \pm 11.6$. In the fusion group, the mean VAS scores for low back pain obtained preoperatively, 6 months after surgery and in the last follow-up visit were $5.5 \pm 2.0,3.2 \pm 1.2$, and $2.8 \pm 1.2$, respectively. The corresponding mean VAS scores for leg pain were $6.0 \pm 2.9,1.7 \pm 0.7$, and $2.0 \pm$ 0.8. The corresponding ODI scores were $63.7 \pm 19.1,32.9 \pm 14.2$, and $30.2 \pm 12.9$. In both groups, VAS $S_{\text {back, leg }}$ and ODI scores decreased after surgery, and there was a statistically significant difference between preoperative and last follow-up scores $(p<0.05)$. There was no statistically significant difference in $\mathrm{VAS}_{\text {back, leg }}$ and ODI scores between the two groups preoperative. However, VAS scores for low back pain at 6 months after surgery and at the last follow-up, ODI scores at the last follow-up were lower in the Dynesys group than that in the fusion group, and the difference was statistically significant.

There was no statistically significant difference in LSDI between the two groups before surgery (21.2 \pm 10.4 versus $23.1 \pm 15.6)$. The LSDI was significantly less in the Dynesys group as compared to the 
fusion group at last follow-up (23.8 \pm 14.2 versus $40.1 \pm 16.4, \mathrm{P} \quad 0.05)$ ( Table 3 ).

\section{Radiological outcomes}

\section{Scoliosis Cobb angle}

In the Dynesys group, the mean scoliosis Cobb angle was $15.1^{\circ} \pm 4.4^{\circ}$ before surgery, $6.9^{\circ} \pm 2.7^{\circ}$ after surgery, and $6.5^{\circ} \pm 2.4^{\circ}$ at the last follow-up. In the fusion group, the mean scoliosis Cobb angle was $16.9^{\circ}$ $\pm 2.6^{\circ}$ before surgery, $6.4^{\circ} \pm 3.7^{\circ}$ after surgery, and $5.2^{\circ} \pm 3.3^{\circ}$ at the last follow-up. Compared with preoperative values, the scoliosis Cobb angle in both groups decreased significantly after surgery and at final follow-up $(P<0.05)$. In the Dynesys group, the difference between postoperative and last follow-up was not statistically significant $(P>0.05)$. There was no statistically significant difference between the two groups in preoperative, postoperative and at last follow-up ( $P$ 0.05) (Table 4).

\section{Lumbar lodorsis}

In the Dynesys group, the mean lumbar lordosis was $31.6^{\circ} \pm 12.5^{\circ}$ before surgery and $36.4^{\circ} \pm 14.8^{\circ}$ after surgery, the difference was statistically significant $(P<0.05)$. At the last follow-up, the figure was $34.1^{\circ}$ $\pm 15.3^{\circ}$, showing no statistical difference compared with that after surgery $(P>0.05)$. In the fusion group, the mean lumbar lordosis was $29.3^{\circ} \pm 7.7^{\circ}$ before operation, and increased to $36.5^{\circ} \pm 11.1^{\circ}$ after operation, with statistically significant difference $(P<0.05)$. At the last follow-up, the figure was $34.8^{\circ} \pm 8.9^{\circ}$, showing no statistical difference compared with that after surgery $(P>0.05)$. There was no statistically significant difference between the two groups in lumbar lordosis before operation, after operation and at the last follow-up $(P>0.05)$ (Table 4).

\section{Range of motion}

ROM values of the implanted segments and L1-S1 levels were measured preoperatively and in the final follow-up. There were no significant differences in the mean ROM values for the implanted segments and the L1-S1 levels between patients in the Dynesys group and fusion group preoperatively $\left(21.9^{\circ} \pm 12.6^{\circ}\right.$ versus $27.3^{\circ} \pm 14.2^{\circ}, P>0.05 ; 36.0^{\circ} \pm 16.3^{\circ}$ versus $\left.28.4^{\circ} \pm 11.6^{\circ}, P>0.05\right)$. However, there were statistical differences in the mean ROM values of the implanted segments and L1-S1 levels between the two groups $(10.7 \pm 5.1$ versus $0.70 \pm 0.66, P<0.05 ; 29.7 \pm 8.0$ versus $8.1 \pm 1.5, P<0.05)$ in the final follow-up (Table 4).

\section{Discussion}

Although scoliosis correction is not the main goal of surgery,it is essential to prevent scoliosis further aggravation. Simotas et al. followed up 49 patients with lumbar spinal stenosis with conservative treatment for 3 years, and found that lumbar scoliosis was one of the reasons for poor results [20]. Instrumented fusion has an advantage in scoliosis correction and maintaining lumbar lordosis. However, there are some disadvantages, such as long operation time, excessive blood loss, and high incidence of perioperative complication[21]. In addition, most patients with lumbar degenerative scoliosis are elderly. 
Surgery less invasive than instrumented fusion should be considered. Therefore, fenestration decompression with selective intervertebral fusion and Dynesys fixation was used in this study. This surgical treatment reduced the operation procedures of the facet joints resection, clearance of intervertebral space and preparation of bone graft bed. Our results indicated that both operation time and blood were significantly less in the Dynesys group compared to fusion. However, the Dynesys group still had an average blood loss of $771 \mathrm{ml}$ during the operation. It is possibly caused by the fenestration decompression in the Dynesys group requiring less bone dissection, which was difficult in patients with degenerative changes, such as hypertrophy of the facet joints and ligamenta flava. Blood loss, operation time, age and the number of instrumented segments are risk factors for perioperative complications [21, 22]. Our data showed the incidence of complications in the Dynesys group was lower than that in the fusion group ( $42.3 \%$ vs. $65 \%$ ), which was most likely due to fewer segments, less blood loss and operation time compared to fusion.

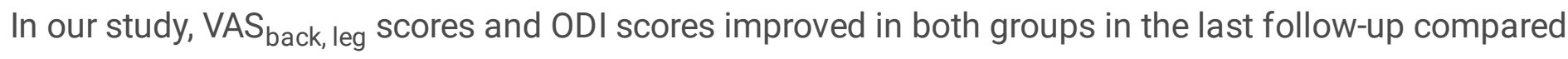
with preoperative scores, indicating that both methods were effective for the treatment of DLS. However, VAS scores for low back pain showed better improvement in the Dynesys group than those obtained in the fusion group, which may be related to Wiltse approach with less disturbance to lumbar dorsal muscles.

In recent years, patients are increasingly concerned with the function of lumbar spine after long segments fusion[23]. Some patients complained about the inconvenience caused by lumbar stiffness in daily life, such as wearing shoes, taking a shower, wiping after stool[24], et al. Robert et al. designed the lumbar stiffness disability index (LSDI) scale to evaluate the limitation of daily activities caused by lumbar stiffness[25]. We used this scale to compare the lumbar function of patients between the two groups. The results showed that the LSDI increased significantly in the fusion group after surgery, while there was no statistical difference between preoperative and last follow-up scores in the Dynesys group. It indicated that dynamic stabilization with the Dynesys system would not impair the function of lumbar spine in treating DLS.

In the Dynesys group, scoliosis was well corrected from $15.1^{\circ}$ preoperatively to $6.9^{\circ}$ postoperatively, and $6.5^{\circ}$ at last follow-up, without significant loss of correction. Although scoliosis correction was better in the fusion group than in the Dynesys group, the difference was not statistically significant.

Lateral olisthesis is an important radiographic parameter that affects the clinical symptoms of patients with DLS. Moderate to severe lateral olisthesis (equal or more than $6 \mathrm{~mm}$ ) demonstrated more severe back pain than mild lateral olisthesis[16]. Intervertebral recurvatum is also an important factor affecting the long-term clinical efficacy. Intervertebral recurvatum or insufficient lordosis causes increased angular motion at the adjacent levels[26]. The loss of lumbar lordosis was also closely related to the clinical symptoms of low back pain[27]. Therefore, significant lateral olisthesis and intervertebral recurvatum should be corrected. However, rigid rods are replaced with elastic spacers made of polycarbonate polyurethane (PCU) and connectors made of polyester fiber (PET) in the Dynesys system, which has 
inadequate ability to correct lateral olisthesis and intervertebral recurvatum. In this study, transforaminal lumbar interbody fusion was performed at the segments with obvious lateral olisthesis and intervertebral recurvatum. In addition, the patients' position was modified to obtain the appropriate lumbar lordosis before stabilization. The results showed that lumbar lordosis were well maintained or improved after operation, and the effect was comparable to that of fusion surgery. Dynesys is unable to correct the obvious sagittal imbalance of the spine, so this study did not involve these cases.

Screw loosening in dynamic stabilization has been a concern. Patients with degenerative scoliosis are mostly elderly, often combined with osteoporosis, making this concern more prominent. In this study, patients with severe osteoporosis were excluded. Only 1 case of screw loosening was found in the Dynesys group at the last follow-up, and no revision surgeries were required. The loosening rate $(1 / 26)$ was lower than that in the fusion group (3/20). One reason may be that the fenestration decompression has less damage to the stability of the lumbar spine. Another cause may be that elastic spacers and connectors can disperse stress on the implants. Yu et al. compared radiographic outcomes of Dynesys and posterior lumbar interbody fusion (PLIF) for the treatment of multisegment degenerative disc disease with a minimum follow-up of 3 years, and confirmed no significant difference in the incidence of screw loosening between the two groups[28]. Wu et al. analyzed 658 screws in 126 patients with an average age of 60.4 years, 31 screws (4.7\%) in 25 patients (19.8\%) were shown to have loosened during an average follow-up period of 37.0 months. All 25 patients with screw loosening were asymptomatic, and in $6(24 \%)$ osseous integration was demonstrated on later follow-up[29]. There were big differences in the incidence of screw loosening with the Dynesys system reported in the literature (range 0-73.5\%), which might be related to the inconsistency of the diagnostic criteria[30]. In our study, the rate of screw loosening was lower compared with previous reports for several reasons. Firstly, the bone and ligament tissue of the posterior column was preserved as much as possible during decompression. Secondly, the screw placement was improved. The pedicle screws with a diameter of $6.4 \mathrm{~mm}$ and a length of $45-$ $50 \mathrm{~mm}$ were inserted on the lateral side of the facet joints as deep as possible. Last, pedicle screws were positioned under imaging control, avoiding reinsertion that might reduce fixation strength.

\section{Conclusions}

This study demonstrated that both Dynesys dynamic stabilization and instrumented fusion can improve clinical outcomes of patients with DLS. Compared to instrumented fusion, fenestration decompression, selective intervertebral fusion and Dynesys stabilizationin have advantages on blood loss, operation time, perioperative complications. In addition, Dynesys stabilization partially preserves the ROM of the stabilized segments that may reduce the limitation on daily activities caused by lumbar stiffness. Dynesys stabilizationin can also correct scoliosis, prevent progression of the curve, and maintain lumbar lordosis in mild to moderate lumbar scoliosis without sagittal imbalance.

\section{List Of Abbreviations}

DLS degenerative lumbar scoliosis 
VAS visual analogue scale

ODI Oswestry disability index

LSDI lumbar stiffness disability index

ROM range of motion

TLIF transforaminal lumbar interbody fusion

SPSS Statistic package for social science

SNK student-newman-keuls

NAIDs non-steroidal anti-inflammatory drugs

PCU polycarbonate polyurethane

PET polyester fiber

PLIF posterior lumbar interbody fusion

\section{Declarations}

Ethics approval and consent to participate

This study has been approved by the Ethical committee of the Third Affiliated Hospital of Chongqing Medical University (Gener Hospital) (SKYW20190106).

Consent for publication

Not applicable.

Availability of data and material

The datasets used and/or analysed during the current study are available from the corresponding author on reasonable request.

Competing interests

The authors declare that they have no competing interests. 
Funding

Not applicable.

Authors' contributions

LL and QZ conceived and designed the study. QZ and FL carried out the operation. $L L, L F, C Z, L C L$ and YHC collected the data. PL and LHL analyzed the data. LL and QZ wrote the manuscript. All authors have read and approved the final manuscript.

Acknowledgements

Not applicable.

\section{References}

1. Birknes JK, White AP, Albert TJ, Shaffrey Cl, Harrop JS. Adult degenerative scoliosis: a review. Neurosurgery. 2008;63:94-103.

2. Xu L, Sun X, Huang S, Zhu Z, Qiao J, Zhu F, et al. Degenerative lumbar scoliosis in Chinese Han population: prevalence and relationship to age, gender, bone mineral density, and body mass index. Eur Spine J. 2013;22:1326-31.

3. Pritchett JW, Bortel DT. Degenerative symptomatic lumbar scoliosis. Spine (Phila Pa 1976). 1993;18:700-3.

4. Ploumis A, Transfledt EE, Denis F. Degenerative lumbar scoliosis associated with spinal stenosis. Spine J. 7:428-36.

5. Simmons ED. Surgical treatment of patients with lumbar spinal stenosis with associated scoliosis. Clin Orthop Relat Res. 2001;45-53.

6. Sengupta DK, Herkowitz HN. Lumbar spinal stenosis. Treatment strategies and indications for surgery. Orthop Clin North Am. 2003;34:281-95.

7. Zhang Z, Ren D, Sun T, Li F, Guan K, Zhao G, et al. Step treatment strategy of degenerative lumbar scoliosis and spinal stenosis. Zhongguo Xiu Fu Chong Jian Wai Ke Za Zhi. 2011;25:951-5.

8. Daubs MD, Lenke LG, Bridwell KH, Cheh G, Kim YJ, Stobbs G. Decompression alone versus decompression with limited fusion for treatment of degenerative lumbar scoliosis in the elderly patient. Evid Based Spine Care J. 2012;3:27-32. 
9. Tsutsui S, Kagotani R, Yamada H, Hashizume H, Minamide A, Nakagawa Y, et al. Can decompression surgery relieve low back pain in patients with lumbar spinal stenosis combined with degenerative lumbar scoliosis? Eur Spine J. 2013;22:2010-4.

10. Cho K-J, Suk S-I, Park S-R, Kim J-H, Kim S-S, Lee T-J, et al. Short fusion versus long fusion for degenerative lumbar scoliosis. Eur Spine J. 2008;17:650-6.

11. Sciubba DM, Scheer JK, Smith JS, Lafage V, Klineberg E, Gupta M, et al. Which daily functions are most affected by stiffness following total lumbar fusion: comparison of upper thoracic and thoracolumbar proximal endpoints. Spine (Phila Pa 1976). 2015;40:1338-44.

12. Di Silvestre M, Lolli F, Bakaloudis G, Parisini P. Dynamic Stabilization for Degenerative Lumbar Scoliosis in Elderly Patients. Spine (Phila Pa 1976). 2010;35:227-34.

13. Di Silvestre M, Lolli F, Greggi T, Vommaro F, Baioni A. Adult's Degenerative Scoliosis: Midterm Results of Dynamic Stabilization without Fusion in Elderly Patients-Is It Effective? Adv Orthop. 2013;2013:365059.

14. Di Silvestre M, Lolli F, Bakaloudis G. Degenerative lumbar scoliosis in elderly patients: Dynamic stabilization without fusion versus posterior instrumented fusion. Spine J. Elsevier Inc; 2014;14:110.

15. Lee S-E, Jahng T-A, Kim H-J. Decompression and nonfusion dynamic stabilization for spinal stenosis with degenerative lumbar scoliosis: Clinical article. J Neurosurg Spine. 2014;21:585-94.

16. Ploumis A, Liu H, Mehbod AA, Transfeldt EE, Winter RB. A correlation of radiographic and functional measurements in adult degenerative scoliosis. Spine (Phila Pa 1976). 2009;34:1581-4.

17. Park WM, Kim CH, Kim YH, Chung CK, Jahng T-A. The Change of Sagittal Alignment of the Lumbar Spine after Dynesys Stabilization and Proposal of a Refinement. J Korean Neurosurg Soc. 2015;58:43-9.

18. Luo L, Zhang C, Zhou Q, Zhao C, Wang L, Liang L, et al. Effectiveness of Transpedicular Dynamic Stabilization in Treating Discogenic Low Back Pain. World Neurosurg. Elsevier Inc; 2018;111:e192-8.

19. Hart RA, Pro SL, Gundle KR, Marshall LM. Lumbar stiffness as a collateral outcome of spinal arthrodesis: a preliminary clinical study. Spine J. 2013;13:150-6.

20. Simotas AC, Dorey FJ, Hansraj KK, Cammisa F. Nonoperative treatment for lumbar spinal stenosis. Clinical and outcome results and a 3-year survivorship analysis. Spine (Phila Pa 1976). 2000;25:197203-4.

21. Cho K-J, Suk S-I, Park S-R, Kim J-H, Kim S-S, Choi W-K, et al. Complications in posterior fusion and instrumentation for degenerative lumbar scoliosis. Spine (Phila Pa 1976). 2007;32:2232-7.

22. Carreon LY, Puno RM, Dimar JR, Glassman SD, Johnson JR. Perioperative complications of posterior lumbar decompression and arthrodesis in older adults. J Bone Joint Surg Am. 2003;85-A:2089-92.

23. Lee CS, Chung SS, Shin SK, Park SJ, Lee HI, Kang KC. Differences in post-operative functional disability and patient satisfaction between patients with long (three levels or more) and short (less than three) lumbar fusions. J Bone Joint Surg Br [Internet]. 2011;93:1400-4. 
24. Bafus T, Shea M, Hart R. Impairment of perineal care functions after long fusions of the lumbar spine. Clin Orthop Relat Res. 2005;111-4.

25. Hart RA, Pro SL, Gundle KR, Marshall LM. Lumbar stiffness as a collateral outcome of spinal arthrodesis: A preliminary clinical study. Spine J. Elsevier Inc; 2013;13:150-6.

26. Akamaru T, Kawahara N, Tim Yoon S, Minamide A, Su Kim K, Tomita K, et al. Adjacent segment motion after a simulated lumbar fusion in different sagittal alignments: a biomechanical analysis. Spine (Phila Pa 1976). 2003;28:1560-6.

27. Chun S-W, Lim C-Y, Kim K, Hwang J, Chung SG. The relationships between low back pain and lumbar Iordosis: a systematic review and meta-analysis. Spine J. 2017;17:1180-91.

28. Yu S-W, Yen C-Y, Wu C-H, Kao F-C, Kao Y-H, Tu Y-K. Radiographic and clinical results of posterior dynamic stabilization for the treatment of multisegment degenerative disc disease with a minimum follow-up of 3 years. Arch Orthop Trauma Surg. 2012;132:583-9.

29. Wu J-C, Huang W-C, Tsai H-W, Ko C-C, Wu C-L, Tu T-H, et al. Pedicle screw loosening in dynamic stabilization: incidence, risk, and outcome in 126 patients. Neurosurg Focus. 2011;31:E9.

30. Pham MH, Mehta VA, Patel NN, Jakoi AM, Hsieh PC, Liu JC, et al. Complications associated with the Dynesys dynamic stabilization system: a comprehensive review of the literature. Neurosurg Focus. 2016;40:E2.

\section{Tables}

Table 1: Inclusion criteria and exclusion criteria 
Inclusion criteria

1. Age $\geq 40$ years at the time of surgery

2. Coronal Cobb angle more than $10^{\circ}$ but less than $20^{\circ}$ before surgery, had an apex between L2 and L4

3. Combined with degenerative changes such as disc herniation, spinal stenosis, spondylolisthesis, etc

4. No improvement afer 3-month conservative treatment

5. Had dynamic fixation (Dynesys system) or instrumented fusion surgery

6. With complete clinical and imaging data

Exclusion criteria

1. Previous history of idiopathic scoliosis and scoliosis caused by tuberculosis, fracture, or other diseases.

2. Previous history of lumbar surgery

3. Sagittal imbalance of the spine

4. Severe osteoporosis ( $\mathrm{T}$ value $\leq-2.5$ with single or multiple fragility fractures or $\mathrm{T}$ value $\leq-3.0$ )

5. With cervical spondylotic myelopathy, hip disease or other diseases that affected the judgment of therapeutic effect

Table 2: Lumbar Stiffness Disability Index[LSDI 
Choose the statement that best describes the effect of low back stiffness on your ability to

1. Bend to your feet to put on your underwear and pants while

dressing independently

2. Bend through your waist to put on your socks and shoes

3. Drive a motor vehicle

4. Perform personal hygiene functions following toileting

5. Bend forward to pick up a small object off the floor 6 .

6. Get in and out of bed

7. Get in and out of a chair

8. Bathe the lower half of your body

9. Get in and out of an automobile

10. Engage in sexual intercourse

Response options and score for each item

0

No effect at all

1 Minor effect

2

Significant effect

3

Require assistance

4

Cannot do at all

LSDI $=$ total score $/(4 \times$ number of questions answered $) \times 100 \%$

\section{Table 3 Clinical outcomes}




\begin{tabular}{lccc}
\hline & Dynesys group $\mathrm{n}=26$ & $\begin{array}{c}\text { Fusion group } \\
\text { Pvalue }\end{array}$ & \\
& & $\mathrm{n}=20$ & \\
\hline VAS back & & & \\
Pre op & $5.2 \pm 1.7$ & $5.5 \pm 2.0$ & 0.58 \\
\hline 6 months postoperative & $2.3 \pm 1.4$ & $3.2 \pm 1.2$ & 0.03 \\
\hline Last follow-up & $2.1 \pm 1.1$ & $2.8 \pm 1.2$ & 0.04 \\
\hline VAS leg & & & \\
\hline Pre op & $5.8 \pm 1.5$ & $6.0 \pm 2.9$ & 0.76 \\
\hline 6 months postoperative & $1.6 \pm 0.4$ & $1.7 \pm 0.7$ & 0.54 \\
\hline Last follow-up & $1.8 \pm 0.9$ & $2.0 \pm 0.8$ & 0.44 \\
\hline ODI (\%) & & & \\
\hline Pre op & $66.9 \pm 17.8$ & $63.7 \pm 19.1$ & 0.56 \\
\hline 6 months postoperative & $30.7 \pm 12.3$ & $32.9 \pm 14.2$ & 0.58 \\
\hline Last follow-up & $21.6 \pm 11.6$ & $30.2 \pm 12.9$ & 0.02 \\
\hline LSDI (\%) & & & \\
\hline Pre op & $21.2 \pm 10.4$ & $23.1 \pm 15.6$ & 0.62 \\
\hline Last follow-up & $23.8 \pm 14.2$ & $40.1 \pm 16.4$ & 0.01 \\
\hline
\end{tabular}

$\mathrm{VAS}_{\text {back }}$ VAS score for back pain, $\mathrm{VAS}_{\text {leg }}$ VAS score for leg pain

\section{Table 4 Radiological outcomes}

\begin{tabular}{|c|c|c|c|}
\hline & Dynesys group $n=26$ & $\begin{array}{c}\text { Fusion group } \\
n=20\end{array}$ & $P$ value \\
\hline $\begin{array}{l}\text { Scoliosis ( ) } \\
\text { Pre op } \\
\text { postoperative } \\
\text { Last follow-up }\end{array}$ & $\begin{array}{c}15.1 \pm 4.4 \\
6.9 \pm 2.7 \\
6.5 \pm 2.4\end{array}$ & $\begin{array}{c}16.9 \pm 2.6 \\
6.4 \pm 3.7 \\
5.2 \pm 3.3\end{array}$ & $\begin{array}{l}0.11 \\
0.60 \\
0.13\end{array}$ \\
\hline $\begin{array}{l}\text { Lumbar lord } \\
\text { Pre op } \\
\text { postoperative } \\
\text { Last follow-up }\end{array}$ & $\begin{array}{r}\text { sis ( , L1-S1) } \\
31.6 \pm 12.5 \\
36.4 \pm 14.8 \\
34.1 \pm 15.3\end{array}$ & $\begin{array}{c}29.3 \pm 7.7 \\
36.5 \pm 11.1 \\
34.8 \pm 8.9\end{array}$ & $\begin{array}{l}0.47 \\
0.98 \\
0.86\end{array}$ \\
\hline $\begin{array}{l}\text { ROM (, L1-S } \\
\text { Pre op } \\
\text { Last follow-up }\end{array}$ & $\begin{array}{c}36.0 \pm 16.3 \\
29.7 \pm 8.0\end{array}$ & $\begin{array}{c}28.4 \pm 11.6 \\
8.1 \pm 1.5\end{array}$ & $\begin{array}{l}0.08 \\
0.01\end{array}$ \\
\hline $\begin{array}{l}\text { ROM (, imp } \\
\text { Pre op } \\
\text { Last follow-up }\end{array}$ & $\begin{array}{c}\text { anted segments) } \\
21.9 \pm 12.6 \\
10.7 \pm 5.1\end{array}$ & $\begin{array}{l}27.3 \pm 14.2 \\
0.70 \pm 0.66\end{array}$ & $\begin{array}{l}0.18 \\
0.01\end{array}$ \\
\hline
\end{tabular}

\section{Figures}




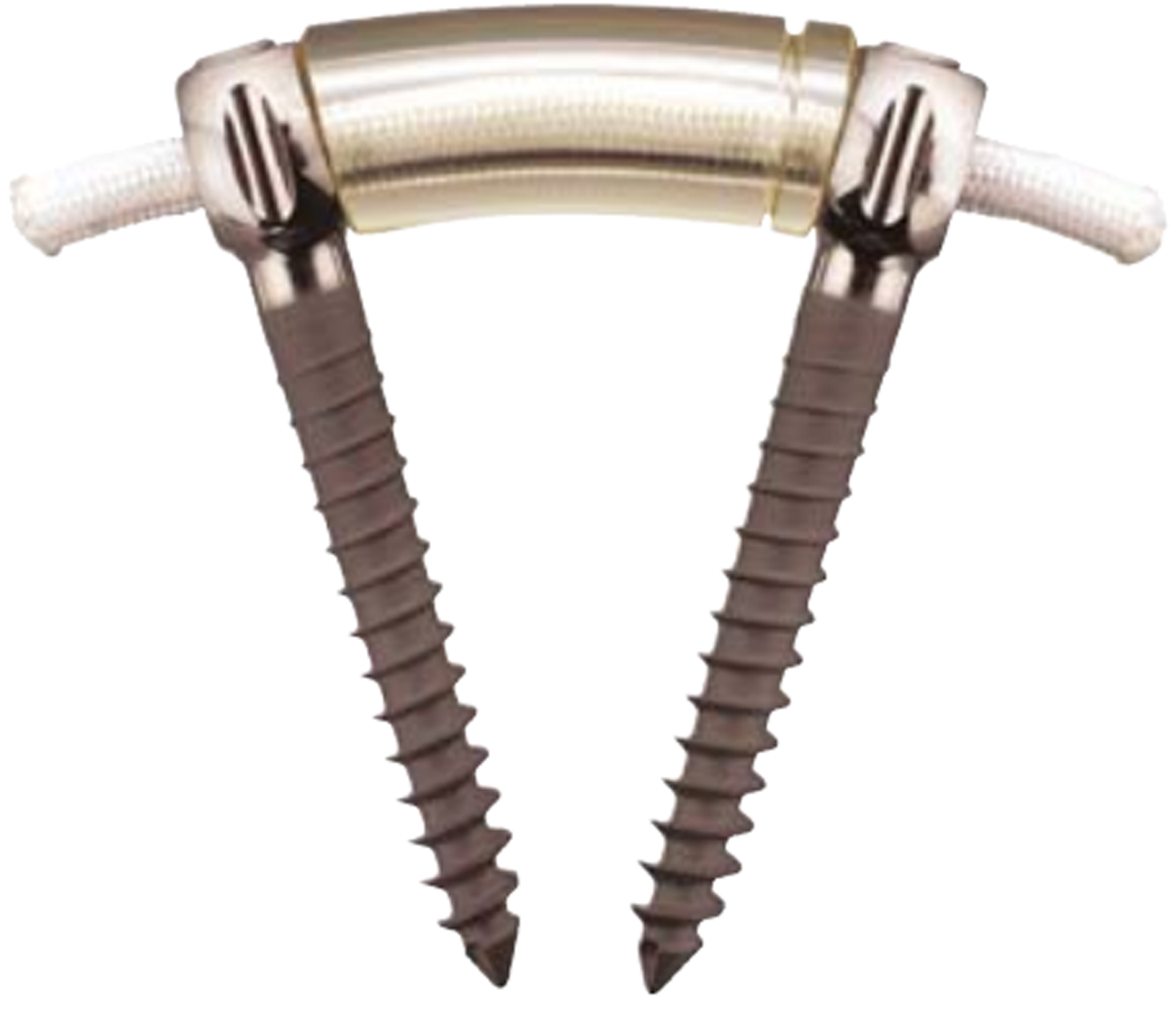

Figure 1

The Dynesys system consists of titaniumaluminum-niobium alloy pedicle screws, polyethylene terephthalate cords, and polycarbonate urethane spacers. The screws are connected with a cord that goes through the center of a hollow cylinder spacer. 

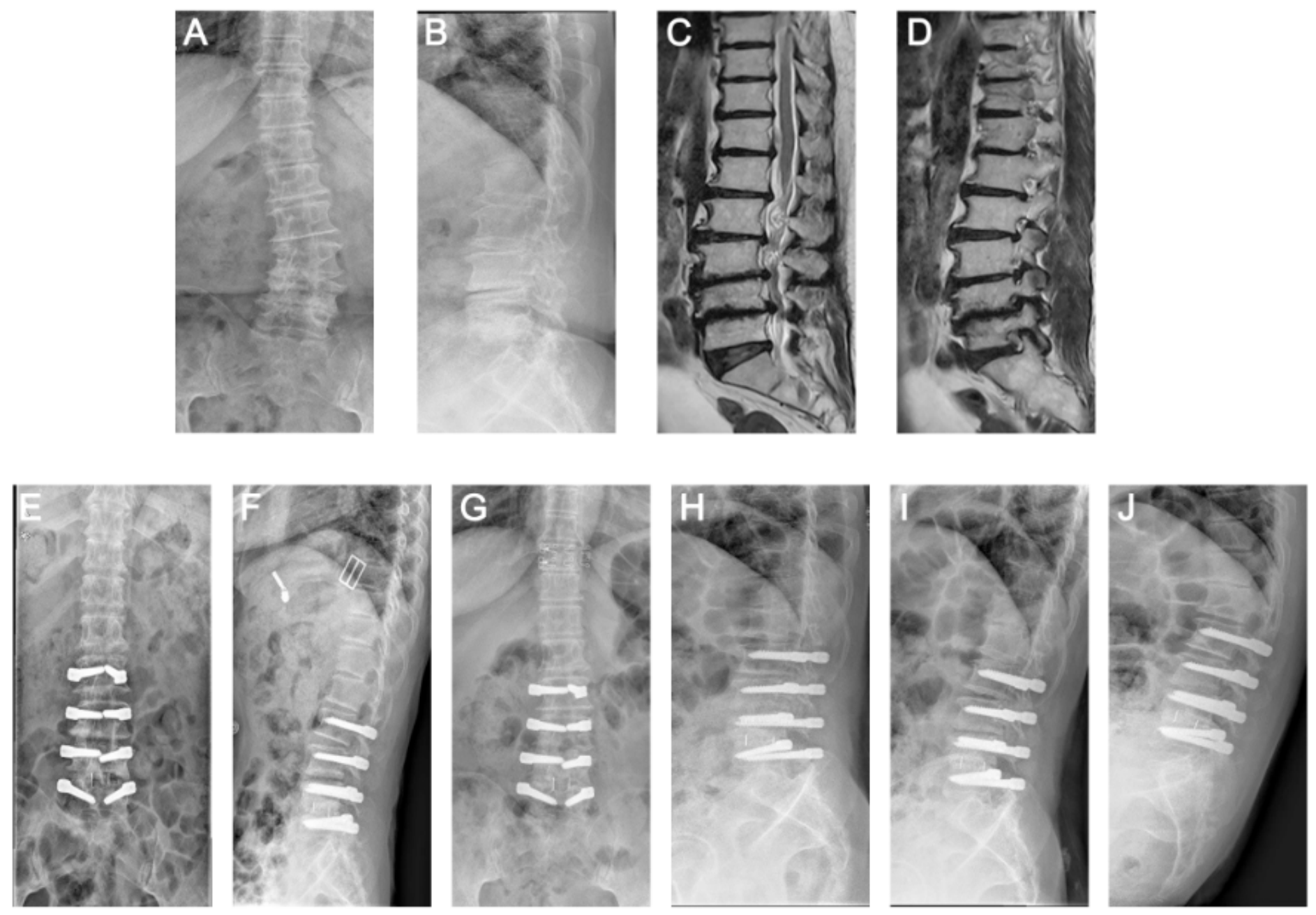

Figure 2

A 49-year-old woman had vertebral canal stenosis at L2 -5 with left lateral recess stenosis at L4-5 and DLS (A-D). She underwent decompressive fenestration at L3-4 with TLIF at L4-5 and instrumentation at L2-5 using the Dynesys system. Postoperative radiographs showed scoliosis correction $(E, F)$, the radiographs obtained 42 months after the operation showed motion preservation and no progression of scoliosis (G-J). 

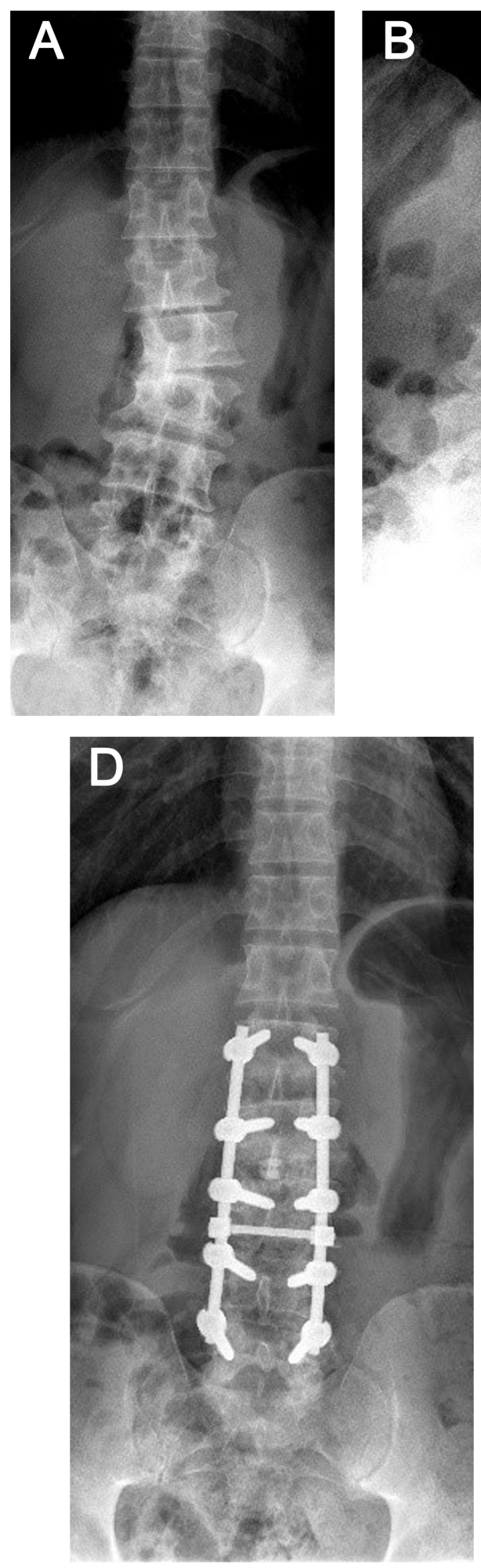
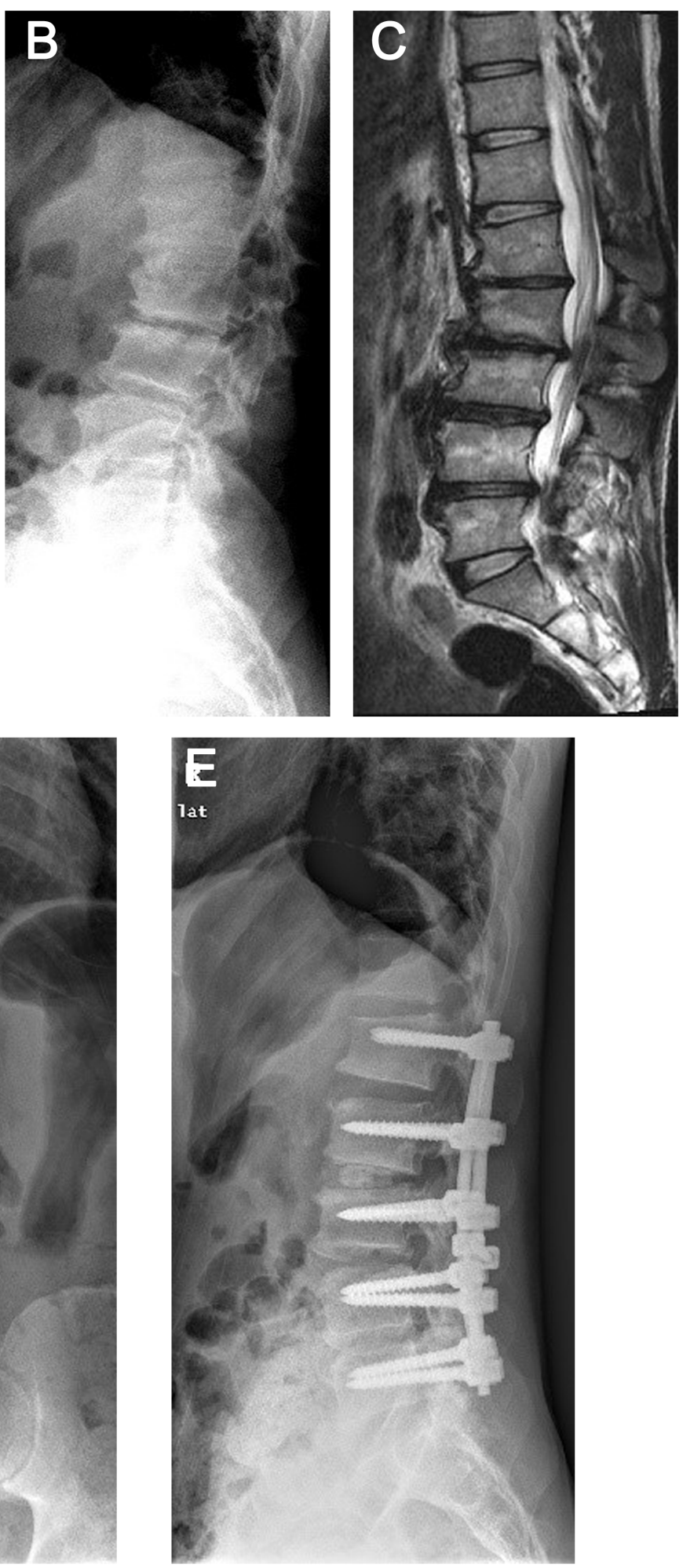

\section{Figure 3}

A 46-year-old man had vertebral canal stenosis at L1-5 and DLS (A-C). She underwent decompressive fenestration at L4-5 with TLIF at L2 -3 and instrumentation at $L 1-5$. The radiographs obtained 38 months after the operation showed stable scoliosis correction (D, E). 